Primary angle closure

\section{Lens extraction in primary angle closure}

\section{W Nolan}

\section{Role debated over many years}

$\mathrm{T}$ he crystalline lens has a pivotal role in primary angle closure (PAC), both in the pathogenesis of pupil block $^{1}$ and by exacerbating the effect of non-pupil block mechanisms such as peripheral iris crowding. Eyes with angle closure tend to have shallow anterior chambers and thick, anteriorly positioned lenses when compared with normal eyes. ${ }^{2-5}$ Removing the lens creates more space in the anterior chamber and widens the angle, which may be enough to achieve intraocular pressure (IOP) control. ${ }^{6}$ The role of lens extraction as a treatment for angle closure has been debated for many years. But with the knowledge that the lens is the single most important contributing factor to the angle closure process, and having acquired the technology and skills to perform relatively safe small incision cataract surgery, should we now be thinking about performing early lens extraction in angle closure patients with the aim of preventing the development of glaucomatous optic neuropathy at a later stage?

In this issue of the BJO (p 14) Tan and co-authors highlight some of the controversies and ethical considerations surrounding the role of early lens extraction in patients with acute angle closure. Theoretically, removing the lens at an early stage will deepen the anterior chamber and open the angle, thus hindering the formation of peripheral anterior synechiae (PAS) and improving the prospects for good long term IOP control. In addition, many of these patients will eventually require surgery for visually significant cataract at some stage. ${ }^{7}$ Tan et al report the corrected visual acuity of patients presenting with acute angle closure soon after resolution of the attack. Just over $50 \%$ of patients obtained a visual acuity of 6/12 or better at a mean interval of 1.7 days after the acute angle closure episode. Factors such as corneal oedema may still be contributing to reduced visual acuity so soon after an attack and one would expect the vision to improve even more over a longer follow up period. The authors think that with this degree of improvement in visual acuity following an acute attack it may not be justifiable to propose primary lens extraction instead of iridotomy as treatment for acute angle closure.

Any discussion of early lens extraction does not in any way imply that ophthalmologists should deviate from current protocols for the management of this potentially blinding condition. All patients presenting with acute angle closure should be treated immediately with systemic and topical medications to lower the IOP, followed by laser iridotomy for the affected and fellow eyes. However, Asian patients who present with acute angle closure can take longer to respond to medical treatment and may require additional interventions such as argon laser peripheral iridoplasty to break the acute attack before performing laser iridotomy. ${ }^{89}$ In the follow up period after an acute attack of angle closure a substantial proportion of Chinese Singaporean subjects develop chronic elevation of IOP and glaucomatous optic nerve damage. ${ }^{7}$ Possible explanations for these findings include delayed initial presentation, ${ }^{10}$ a greater role for nonpupil block mechanisms in Asian patients with PAC and the presence of pre-existing asymptomatic primary angle closure glaucoma (PACG) before the acute attack.

If we are to attempt to implement prevention of blindness programmes targeted at primary angle closure glaucoma we need evidence that our interventions are effective in preventing disease progression and visual loss

The trial being run by this group in Singapore comparing primary lens extraction with iridotomy in acute angle closure will help determine whether early surgical intervention can benefit the patient by reducing their need for future medical or surgical glaucoma treatment. Until this has been proved, lens extraction, which is often technically demanding and runs the risk of complications when performed in these eyes, should be reserved for cases in which the acute attack is not responding to conventional medical and laser treatment. In these cases removal of the lens is often an effective means of achieving rapid control of the IOP. ${ }^{11}$

When faced with a patient presenting with acute symptomatic primary angle closure it is difficult to make judgments on how much the lens is contributing to the disease. Assessment of the contribution of lens opacity to poor visual acuity is complicated by the presence of corneal oedema and it is often difficult to obtain a clear gonioscopic view of the angle. In the setting of asymptomatic PAC the picture is less complicated, there is more time to consider the different management options, and the surgery is technically more straightforward. However, the role of lens extraction in this form of the disease is still unclear and there is currently little in the way of evidence to guide us.

For a patient who has residual appositional angle closure following iridotomy and coexisting lens opacity, it is reasonable to have a low threshold for doing cataract surgery at the earliest sign of visual symptoms. Difficulties arise when dealing with cases in which the lens appears to be making a significant contribution to the residual angle closure but there is no significant cataract and visual acuity is good. Does this situation justify a clear lens extraction and can prophylactic surgery prevent future development of PACG in these cases? Angle imaging methods, such as ultrasound biomicroscopy and anterior segment optical coherence tomography, can be useful tools in determining mechanisms underlying post-iridotomy angle closure and guiding the clinician towards the appropriate treatment. Eyes in which imaging demonstrates anterior rotation of ciliary processes and plateau iris configuration may respond to argon laser peripheral iridoplasty..$^{12}$ Otherwise, in cases in which IOP is normal and there are no signs of optic nerve head damage the patient can probably be observed.

The other area of difficulty is in deciding whether cataract surgery alone can control the IOP in more established disease with glaucomatous optic neuropathy, or whether it needs to be combined with trabeculectomy. Some studies suggest that cataract surgery may be as effective as filtering surgery in controlling IOP in PACG cases. ${ }^{14} 15$ But it may be that stage and chronicity of the angle closure process dictate which surgery should be done to achieve optimum outcomes. In cases in which there is early optic disc cupping and mild visual field loss, lens extraction alone may be enough to achieve 
adequate IOP control; whereas eyes with advanced glaucomatous optic neuropathy are more likely to have poor residual trabecular meshwork function as a result of PAS or non-synechial damage. ${ }^{16}$ In such cases phacotrabeculectomy may be necessary to achieve the degree of IOP control required to prevent progression of glaucomatous optic neuropathy. This is a similar theory to that used to explain why laser iridotomy appears to be less effective in controlling IOP in advanced PACG. ${ }^{17-19}$ It is probably oversimplifying things to extrapolate data from laser studies to the surgical management of PACG and other issues need to be considered. These include the frequency and consequences of IOP spikes following cataract surgery in angle closure patients and whether target pressures aimed for following surgery in POAG patients should be applied to patients with PACG. Studies investigating the effectiveness of surgical interventions for angle closure should be designed with these factors in mind.

A randomised controlled trial is under way in Hong Kong comparing phacoemulsification with phacotrabeculectomy for PACG (CC Tham, personal communication). The results of this and other ongoing trials in Asia investigating the effectiveness of early detection and treatment for primary angle closure are needed to help guide clinicians when making decisions on which interventions are likely to be beneficial to the patient. From a public health perspective PACG has been projected to be one of the commonest causes of irreversible blindness in the populous countries of Asia.$^{20}$ If we are to attempt to implement prevention of blindness programmes targeted at PACG we need evidence that our interventions are effective in preventing disease progression and visual loss.

Br J Ophthalmol 2006;90:1-2.

doi: 10.1136/bjo.2005.082040

Correspondence to: Dr Winifred P Nolan, Department of Ophthalmology, National University Hospital, 5 Lower Kent Ridge Road, Singapore 138686; winnie_nolan@yahoo.com

\section{REFERENCES}

1 Tiedeman JS. A physical analysis of the factors that determine the contour of the iris. Am J Ophthalmol 1990;111:338-43.

2 Lowe RF. Aetiology of the anatomical basis for primary angle-closure glaucoma. $\mathrm{Br} J$ Ophthalmol 1970;54:161-9.

3 Törnquist R. Shallow anterior chamber in acute angle-closure. A clinical and genetic study. Acta Ophthalmol 1953;31(Suppl 39):1-74.

4 Alsbirk PH. Anterior chamber depth and primary angle-closure glaucoma. I. An epidemiologic study in Greenland Eskimos. Acta Ophthalmol 1975:53:89-104.

5 George R, Paul PG, Baskaran M, et al. Ocular biometry in occludable angles and angle closure glaucoma: a population based survey Br J Ophthalmol 2003:87:399-402.

6 Hayashi K, Hayashi H, Nakao F, et al. Changes in anterior chamber angle width and depth after intraocular lens implantation in eyes with glaucoma. Ophthalmology 2000;107:698-703.

7 Aung T, Friedman DS, Chew PT, et al. Long-term outcomes in Asians after acute primary angle closure. Ophthalmology 2004;111:1464-9.
8 Tham CC, Lai JS, Leung DY, et al. Acute primary angle closure. Ophthalmology 2005; 112:1479-80.

9 Lam DS, Lai JS, Tham CC, et al. Argon laser peripheral iridoplasty versus conventional systemic medical therapy in treatment of acute primary angle-closure glaucoma: a prospective, randomized, controlled trial. Ophthalmology 2002;109:1591-6.

10 Seah SK, Foster PJ, Chew PT, et al. Incidence of acute primary angle-closure glaucoma in Singapore. An island-wide survey. Arch Ophthalmol 1997;115:1436-40.

11 Roberts TV, Francis IC, Lertusumitkul S, et al. Primary phaocemulsification for uncontrolled angle-closure glaucoma. J Cataract Refract Surg 2000;26:1012-16.

12 Chew PTK, Yeo LMW. Argon laser iridoplasty in chronic angle closure glaucoma. Int Ophthalmol 1995; 19:67-70.

13 Ritch R, Tham CCY, Lam DSC. Long-term success of argon laser peripheral iridoplasty in the management of plateau iris syndrome. Ophthalmology 2004;111:104-8.

14 Greve EL. Primary angle closure glaucoma: extracapsular cataract extraction or filtering procedure. Int Ophthalmol 1988; 12:157-62

15 Gunning FP, Greve EL. Lens extraction for uncontrolled angle-closure glaucoma: long-term follow-up. J Cataract Refract Surg 1998;24:1347-56.

16 Sihota R, Lakshmaiah NC, Walia KB, et al. The trabecular meshwork in acute and chronic angle closure glaucoma. Indian J Ophthalmol 2001:49:255-9.

17 Salmon JF. Long-term intraocular pressure control after Nd-YAG laser iridotomy in chronic angle-closure glaucoma. J Glaucoma 1993;2:291-6.

18 Kim YY, Jung HR. Dilated miotic-resistan pupil and laser iridotomy in primary angleclosure glaucoma. Ophthalmologica 1997;211:205-8.

19 Nolan WP, Foster PJ, Devereux JG, et al. YAG laser iridotomy treatment for primary angle closure in east Asian eyes. $\mathrm{Br} J$ Ophthalmol 2000;84: 1255-9.

20 Foster PJ, Johnson GJ. Glaucoma in China: how big is the problem? $\mathrm{Br} J$ Ophthalmol 2001;85:1271-2.

\section{Prevention of diabetic blindness}

\section{E Stefánsson}

\section{New technologies or "old fashioned" public health?}

W ophthalmologists know how to prevent diabetic blindness, but we are not doing it. The scientific principles of treatment of diabetic retinopathy and prevention of blindness have been known for over 20 years. In spite of this, diabetic eye disease remains a major public health problem with large numbers of people with diabetes going blind worldwide from what is largely a preventable cause of blindness. ${ }^{1-6}$ The problem will expand rapidly in the decades to come with the ongoing worldwide epidemic of type 2 diabetes mellitus. ${ }^{7}$ Is it possible that our efforts in this field are directed too much towards new inventions in diagnostic technologies and treatment and not enough towards old fashioned public health efforts and health care, using the equipment and knowledge we already have?

Specific treatment for diabetic retinopathy was initially limited to pituitary gland destruction. In the 1970s this was replaced with photocoagulation, and the Diabetic Retinopathy Study ${ }^{8}$ confirmed the benefit of xenon arc or argon laser photocoagulation to reduce the risk of visual loss in people with diabetes with proliferative retinopathy. A few years later the Early Treatment Diabetic Retinopathy Study ${ }^{9}$ confirmed the utility of macular laser photocoagulation to reduce the risk of visual loss in patients with diabetic macular oedema. In both proliferative diabetic retinopathy and diabetic macular oedema the benefit of laser treatment is critically related to the timing of the treatment. The treatment is highly effective when applied in the early stages of proliferative retinopathy or diabetic macular oedema but less effective and more difficult if the disease is more advanced. The use of laser treatment in diabetic eye disease has revolutionised the treatment of diabetic eye disease and probably millions of diabetic patients have been saved from severe vision loss with this treatment.

While this has been extremely beneficial in many individual cases, a different picture emerges if the situation is examined from a public health viewpoint. Diabetic eye disease remains a major cause of blindness in the world, also in some of the richest societies. ${ }^{4} 10$ 
The public health failure is not universal. Systematic screening programmes for diabetic eye disease and preventive treatment have been organised in some regions and the outcome has been documented. ${ }^{11}$ The longest experience is in Iceland where systematic screening for diabetic eye disease has been in place for 25 years. In 1980 2.4\% of Icelandic people with diabetes were legally blind (visual acuity $<0.1$ ) and in 2005 this number is $0.5 \%$. This has been achieved with a "low tech" public health approach. ${ }^{12}{ }^{13}$ Similar benefit from a public health approach to diabetic eye disease has been seen in a few other places in northern Europe. In each instance the prevalence of diabetic blindness has gone down and incidence studies have shown that the annual incidence of diabetic blindness can be brought down to $1 \%$ or less. ${ }^{14}{ }^{15}$ This is in sharp contrast with surveys from areas where a public health approach with systematic screening and preventive treatment has not been in place. For example, in Wisconsin, Klein et $a l^{16}$ have reported $3.6 \%$ prevalence of legal blindness among people with diabetes and $4.6 \%$ with partial sight, and Jerneld and Algvere $^{17}$ reported $7.7 \%$ legal blindness and $9.3 \%$ partial sight in a Swedish population that was not being screened for diabetic retinopathy in the 1980s.

The pressing need is for a public health approach using present technology rather than the development of new technologies

The standard of treatment and prevention is universally accepted. The World Health Organization and many professional organisations recommend yearly fundus examination of diabetic patients and preventive treatment as indicated by the DRS (Diabetic Retinopathy Study) and ETDRS (Early Treatment Diabetic Retinopathy Study) studies. ${ }^{18}$ The fact remains that these standards of treatment are not generally followed. Campaigns organised to improve this situation such as the Diabetes 2000 program of the American Academy of Ophthalmology and the St Vincent Declaration in Europe have helped, but have not been able to solve the public health problem.

If diabetic blindness can be prevented in Nordic communities of a few hundred thousand inhabitants, there should be no reason why it cannot be replicated in larger communities and around the world. Any community willing to invest in diabetic eye screening can expect the number of legally blind diabetic patients to decrease by twofold to threefold within 10 years and decrease the disability expenditures by an amount that is many times the initial investment. ${ }^{19} 20$

Ideally, prevention of diabetic blindness would be supported by efforts to prevent diabetic retinopathy through optimal treatment of blood sugar and blood pressure levels and, ultimately, by the prevention of type 2 diabetes with public and education correction of the lifestyle that leads to obesity and diabetes. $^{21}$ This, however, may be outside the scope of ophthalmology. On the other hand, the prevention of diabetic blindness is very much the duty of ophthalmologists and the public health failure in dealing with it puts the world's ophthalmological community to shame.

\section{IS THERE ANY VALUE IN EARLY DETECTION OF DIABETIC RETINOPATHY?}

A considerable research effort is being made in diabetic eye disease. New and older drugs are being studied that may help treat diabetic eye disease ${ }^{4}$ and a number of scientists are studying new technologies to detect and diagnose diabetic retinopathy. These techniques include fluorophotometry and fluorescein angiography and electroretinography, and this issue of the BJO ( $p$ 17) contains an elegant study by El-Bradey et al on scanning laser entoptic perimetry for the early detection of visual defects associated with diabetic retinopathy. It is clear that these techniques are able to detect early diabetic retinopathy and even detect changes in the retina before diabetic retinopathy changes are visible by fundus examination. But what is the value of detecting diabetic change in the retina at this early stage?

In the present clinical situation there is no clinical value in the detection of diabetic retinal disease before the occurrence of microaneurysms. The detection of mild non-proliferative retinopathy also has very little clinical value, in that no treatment would be instituted and the patient would receive the same general advice regarding blood glucose and blood pressure control. ${ }^{21}$ It is only the detection of early macular oedema or neovascularisation that would call for specific treatment. This usually takes place rather late in the development of diabetic retinopathy and is easily detectable by biomicroscopy at the slit lamp.

Patients with diabetes are not going blind for lack of technology or treatment options. They are going blind because they are not receiving treatment that has been well established for more than a quarter of century. The pressing need is for a public health approach using present technology rather than the development of new technologies.
Br J Ophthalmol 2006;90:2-3.

doi: 10.1136/bjo.2005.082065

Correspondence to: Professor Einar Stefánsson, University of Iceland, Landspitali Hringbraut, Reykjavik 110, Iceland; einarste@landspitali.is

\section{REFERENCES}

1 Kahn HA, Hiller R. Blindness caused by diabetic retinopathy. Am J Ophthalmo 1974;78:58-67.

2 Sorsby A. The incidence and causes of blindness in England and Wales 1963-68. Department of Health and Social Security. Reports on Public Health and Medical Subjects, No 128. With an appendix on services available for incipient blindness. Rep Public Health Med Subj (Lond) 1972;0:1-72.

3 Ghafour IM, Allan D, Foulds WS. Common causes of blindness and visual handicap in the west of Scotland. Br J Ophthalmol 1983;67:209-13.

4 Porta M, Allione A. Current approaches and perspectives in the medical treatment of diabetic retinopathy. Pharmacol Ther 2004; 103:167-77.

5 Trautner C, Icks A, Haastert B, et al. Incidence of blindness in relation to diabetes. A population-based study. Diabetes Care 1997;20:1147-53.

6 Broadbent DM, Scott JA, Vora JP, Harding SP. Prevalence of diabetic eye disease in an inner city population: the Liverpool Diabetic Eye Study. Eye 1999:13(P+ 2): 160-5

7 Zimmet P, Alberti KG, Shaw J. Global and societal implications of the diabetes epidemic Nature 2001;414:782-7.

8 TDRSR Group. Photocoagulation treatment of proliferative diabetic retinopathy. Clinical application of Diabetic Retinopathy Study (DRS) findings, DRS Report Number 8. Ophthalmology $1981 ; 88: 583-600$

9 Early Treatment Diabetic Retinopathy Study Research Group. Photocoagulation for diabetic macular edema. Early Treatment Diabetic Retinopathy Study report number 1. Arch Ophthalmol 1985:103:1796-806.

10 Munier A, Gunning T, Kenny D, et al. Causes of blindness in the adult population of the Republic of Ireland. Br J Ophthalmol 1998;82:630-3

11 Younis N, Broadbent DM, James M, et al. Current status of screening for diabetic retinopathy in the UK. Diabet Med 2002;19(Suppl 4):44-9.

12 Stefansson E, Bek T, Porta M, et al. Screening and prevention of diabetic blindness. Acta Ophthalmol Scand 2000:78:374-85.

13 Zoega GM, Gunnarsdottir T, Biörnsdóttir S, et al. Screening compliance and visual outcome in diabetes. Acta Ophthalmol Scand (in press).

14 Agardh E, Agardh CD, Hansson-Lundblad C. The five-year incidence of blindness after introducing a screening programme for early detection of treatable diabetic retinopathy. Diabet Med 1993;10:555-9

15 Kristinsson JK. Diabetic retinopathy. Screening and prevention of blindness. A doctoral thesis. Acta Ophthalmol Scand (Suppl) 1997: 1-76.

16 Klein R, Klein BE, Moss SE. Visual impairment in diabetes. Ophthalmology 1984:91:1-9.

17 Jerneld B, Algvere P. Visual acuity in a diabetic population. Acta Ophthalmol 1987;65:170-7.

18 Harding S, Greenwood R, Aldington S, et al. Grading and disease management in national screening for diabetic retinopathy in England and Wales. Diabet Med 2003:20:965-71.

19 Javitt JC, Canner JK, Sommer A. Cost effectiveness of current approaches to the control of retinopathy in type I diabetics. Ophthalmology 1989;96:255-64.

20 James M, Turner DA, Broadbent DM, et al. Cost effectiveness analysis of screening for sight threatening diabetic eye disease. BMJ 2000;320:1627-31.

21 Stratton IM, Kohner EM, Aldington SJ, et al. UKPDS 50: risk factors for incidence and progression of retinopathy in type II diabetes over 6 years from diagnosis. Diabetologia 2001;44:156-63. 
Trabeculectomy

\section{Building a safer trabeculectomy}

$S$ Lin

\section{A new approach to preventing hypotony and shallow/flat anterior chamber}

I $\mathrm{n}$ this issue of the BJO ( $\mathrm{p}$ 44), Stalmans et al describe their clinical results of a new technique for trabeculectomy, originally described by Wells et al. ${ }^{1}$ In this modification of the traditional trabeculectomy procedure, there are three main alterations: (1) adjustable/removable sutures are placed along each lateral side of the trabeculectomy flap for intraoperative and postoperative adjustment; (2) an anterior chamber maintainer is placed to titrate the leakage from the trabeculectomy and to wash out inflammatory debris from the anterior chamber; and (3) a standardised excision is created using the Khaw punch instrument of $0.5 \mathrm{~mm}$. The overall purported advantage of these changes is reduced complications related to early postoperative overfiltration.

The modern Cairns/Watson technique for trabeculectomy ${ }^{2}{ }^{3}$ was developed, in part, to avoid the overfiltration associated with full thickness sclerostomies. Clinical studies have demonstrated lower rates of hypotony and flat chamber with the guarded filtration approach. ${ }^{4}$ Subsequent progress with the use of antimetabolites, such as mitomycin C (MMC) and 5-fluorouracil (5-FU), have extended the efficacy of trabeculectomy procedures. ${ }^{5}$ However, these agents have also led to an increased incidence of some complications, including chronic hypotony and bleb leakage..$^{5-8}$

Despite the improvements compared with full thickness filters, trabeculectomy remains a surgery associated with relatively high rates of complications (including hypotony and shallow/flat chamber), a substantial postoperative recovery period, and significant long term failure rates. ${ }^{5-8}$ These factors have fuelled the search for safer and more effective outflow procedures. In particular, newer surgeries have been developed to avoid the hypotony and need for bleb formation. Non-penetrating surgeries such as viscocanalostomy ${ }^{9}$ and deep sclerectomy ${ }^{10}$ (with or without collagen implant) do not create an entry into the anterior chamber, and result in minimal to no bleb formation. Although they have been shown to be less effective for intraocular pressure (IOP) control than trabeculectomies, ${ }^{11}{ }^{12}$ they remain attractive procedures, the result in large part of their lower rates of hypotony, shallow/flat chambers, and other complications. ${ }^{11}{ }^{12}$ Other recent surgical advancements include the development of valved tube shunts ${ }^{13}$ and the Trabectome, ${ }^{14}$ both of which theoretically avoid hypotony, as well as bleb formation in the case of Trabectome. Future prospective, randomised trials will shed light on the effectiveness and rate of complications of these procedures compared to trabeculectomy.

Results of this novel trabeculectomy technique appear favourable in terms of final IOP outcome and low or medium rates of serious complications

Although these alternative outflow procedures have gained greater use, trabeculectomy remains the gold standard filtering surgery. Past improvements in technique have included releasable sutures ${ }^{15}$ and small incision trabeculectomy. ${ }^{16}$ In this issue, clinical results of a modified technique for trabeculectomy are presented. Wells et al originally described the modifications and their results obtained in donor eyes. ${ }^{1}$ Using real time IOP monitoring, they found that manipulation of the adjustable/releasable sutures was more effective and safer than massage of the posterior lip or removal of the adjustable/releasable sutures. In addition, suture adjustment led to a more rapid stabilisation of IOP, so that the resultant stable IOP could be checked in the office soon afterwards. The actual trabeculectomy orifice was standardised using the Khaw punch of $0.5 \mathrm{~mm}$. Additional reported advantages relate to the use of an anterior chamber maintainer during surgery and include reduced risk of scarring (from the "washout" of inflammatory debris) and complications resulting from IOP fluctuation and anterior chamber collapse.

In their retrospective study of 56 eyes of 53 patients treated by this modified trabeculectomy technique, Stalmans et al report a drop in IOP from a mean of $21.2 \mathrm{~mm} \mathrm{Hg}$ preoperatively to $12.8 \mathrm{mmHg}$ postoperatively, with a mean follow up of 15.7 months. All of the eyes had an IOP $<21 \mathrm{~mm} \mathrm{Hg}$ and $80 \%$ had IOP $<18 \mathrm{~mm} \mathrm{Hg}$ at last follow up. Furthermore, there appears to be relatively low or comparable rates of complications: $2 \%$ flat anterior chamber requiring surgery, $1.5 \%$ hypotony $>3$ weeks, 9\% choroidal detachment, $5 \%$ hyphaema, $1.5 \%$ malignant glaucoma, and $1.5 \%$ endophthalmitis.

A significant concern with the clinical findings of this new surgical approachwhose major aim is to avoid consequences of hypotony and flat chamberis the relatively "high" mean IOP in the early postoperative period, a factor that may be associated with a greater risk for follow up interventions and late IOP rise. ${ }^{17-19}$ On postoperative day 1 , the mean IOP was $10.4 \mathrm{~mm} \mathrm{Hg}$ with a range of $2-30 \mathrm{~mm} \mathrm{Hg}$. With the standard trabeculectomy technique, the desired appearance for day 1 is an IOP in the 5-10 $\mathrm{mm} \mathrm{Hg}$ range with a formed anterior chamber. ${ }^{11} 20$ The IOP usually rises from this initial "low" pressure, and additional interventions may be required to achieve or maintain an acceptable IOP. In their report on a series of combined trabeculectomy and cataract extraction surgeries, Morris et $a l^{17}$ have shown that an IOP $>14 \mathrm{~mm} \mathrm{Hg}$ in the first operative week was a significant independent risk factor for early filtration failure requiring suture release, and was associated with a lower success rate at 12 months and 24 months. Results from the Advanced Glaucoma Intervention Study ${ }^{18}$ as well as Porges and Ophir ${ }^{19}$ also suggest that early IOP rise is correlated with a higher risk of late failure and need for topical glaucoma medications.

There are some indications of early IOP rise and need for postoperative adjustments and interventions in the present paper. The authors report a high rate of suture manipulations in the first month, with $38 \%$ requiring argon suture lysis of releasable sutures, $5 \%$ receiving loosening of adjustable sutures, and $32 \%$ needing removal of adjustable sutures. Such interventions are perhaps expected consequences of this modified technique and are relatively non-invasive. However, surgical intervention in the form of needling of the bleb was necessary in $14 \%$ of eyes with a cystic bleb and $18 \%$ of eyes with a nonfunctional bleb. This $32 \%$ rate of needling is high compared to that found in the National Survey of Trabeculectomy $^{21}(3.7 \%)$, and may represent a form of "failure" among those requiring needling. 
Although the title of the study includes the designation of "long term outcome," a mean follow up period of 15.7 months may represent intermediate follow up, given the cumulative risk for failure with each passing year after trabeculectomy. The success at 2 years, 3 years, and subsequent years will be of particular interest to see if the comparatively lower aqueous flow in the immediate postoperative period has long term consequences. In addition, a statistical evaluation of the current data to correlate the initial postoperative IOPs with later IOPs and the need for needling revision would be instructive.

In terms of avoidance of complications related to hypotony, there were no cases of chronic hypotony and only one case of flat chamber that required surgery. However, the incidence of choroidal detachment was $9 \%$, suggesting that some of the patients may have had early hypotony and/or shallow chamber. These latter data are not presented in the paper. Choroidal haemorrhage did not occur in the present series but is a rare complication. Among the 525 patients who received trabeculectomy in the Collaborative Initial Glaucoma Treatment Study, none experienced expulsive choroidal haemorrhage. ${ }^{22}$

The other suggested benefit in the modified trabeculectomy is the reduction of postoperative inflammation and fibrosis as a result of using the anterior chamber maintainer to wash out debris during the operation. Results are not presented on the grade of postoperative inflammation, however, which might have been supportive of this issue. The effects on scarring are best judged by the need for follow up interventions, which seemed significant, as discussed above.
In summary, the clinical results of this novel trabeculectomy technique appear favourable in terms of final IOP outcome and low or medium rates of serious complications, although postoperative suture adjustments and bleb needling are required in a substantial number of patients. Ultimately, to compare the results more directly with the standard trabeculectomy technique will require a large prospective, randomised trial.

Br J Ophthalmol 2006;90:4-5.

doi: $10.1136 /$ bjo.2005.078568

Correspondence to: S Lin, Department of Ophthalmology, University of California, San Francisco, 10 Koret Way, San Francisco, CA 94143, USA; lins@vision.ucsf.edu

Competing interest statement: The author has no competing interests related to any of the devices or drugs discussed in this publication.

\section{REFERENCES}

1 Wells AP, Bunce C, Khaw PT. Flap and suture manipulation after trabeculectomy with adjustable sutures: titration of flow and intraocular pressure in guarded filtration surgery. J Glaucoma 2004;13:400-16.

2 Cairns JE. Trabeculectomy. Preliminary report of a new method. Am J Ophthalmol 1968:66:673-9.

3 Watson PG. Trabeculectomy: a modified ab externo technique. Ann Ophthalmol 1970;2:199-205.

4 Borisuth NS, Phillips B, Krupin T. The risk profile of glaucoma filtration surgery. Curr Opin Ophthalmol 1999;10:112-16.

5 Wormald R, Wilkins MR, Bunce C. Post-operative 5 -fluorouracil for glaucoma surgery. Cochrane Database Syst Rev 2001;CD001132.

6 Wilkins M, Indar A, Wormald R. Intra-operative mitomycin C for glaucoma surgery. Cochrane Database Syst Rev 2001;CD002897. Review.

7 Kim YY, Sexton RM, Shin D, et al. Outcomes of primary phakic trabeculectomies without versus with 0.5 to 1 -minute versus $3-5$ minute mitomycin C. Am J Ophthalmol 1998;126:755-62.

8 Bindlish R, Condon GP, Schlosser JD, et al. Efficacy and safety of mitomycin-C in primary trabeculectomy: five-year follow-up. Ophthalmology 2002;109:1336-41
9 Stegmann R, Pienaar A, Miller D. Viscocanalostomy for open-angle glaucoma in black African patients. J Cataract Refract Surg 1999:25:316-22.

10 Lachkar Y, Neverauskiene J, Jeanteur-Lunel MN, et al. Nonpenetrating deep sclerectomy: a 6-year retrospective study. Eur $J$ Ophthalmol 2004;14:26-36.

11 O'Brart DP, Shiew M, Edmunds B. A randomised, prospective study comparing trabeculectomy with viscocanalostomy with adjunctive antimetabolite usage for the management of open angle glaucoma uncontrolled by medical therapy. $\mathrm{Br} J$ Ophthalmol 2004;88:1012-17.

12 Chiselita D. Non-penetrating deep sclerectomy versus trabeculectomy in primary open-angle glaucoma surgery. Eye 2001;15(Pt 2):197-201.

13 Topouzis F, Coleman AL, Choplin N, et al. Follow-up of the original cohort with the Ahmed glaucoma valve implant. Am J Ophthalmol 1999; 128:198-204.

14 Minckler DS, Baerveldt G, Alfaro MR, et al. Clinical results with the Trabectome for treatment of open-angle glaucoma. Ophthalmology 2005; 1 12:962-7.

15 Kolker AE, Kass MA, Rait JL. Trabeculectomy with releasable sutures. Arch Ophthalmol 1994; 112:62-6.

16 Lerner SF. Small incision trabeculectomy avoiding Tenon's capsule. A new procedure for glaucoma surgery. Ophthalmology 1997:104:1237-41.

17 Morris DA, Peracha MO, Shin DH, et al. Risk factors for early filtration failure requiring suture release after primary glaucoma triple procedure with adjunctive mitomycin. Arch Ophthalmol 1999:117:1149-54.

18 The Advanced Glaucoma Intervention Study (AGIS): 11. Risk factors for failure of trabeculectomy and argon laser trabeculoplasty. Am J Ophthalmol 2002;134:481-98.

19 Porges Y, Ophir A. Surgical outcome after early intraocular pressure elevation following combined cataract extraction and trabeculectomy. Ophthalmic Surg Lasers 1999:30:727-33.

20 Batterbury M, Wishart PK. Is high initial aqueous outflow of benefit in trabeculectomy? Eye 1993; 7(Pt 1): 109-12.

21 Edmunds B, Thompson JR, Salmon JF, et al. The National Survey of Trabeculectomy. III. Early and late complications. Eye 2002;16:297-303.

22 Lichter PR, Musch DC, Gillespie BW, et al. Interim clinical outcomes in the Collaborative Initial Glaucoma Treatment Study comparing initial treatment randomized to medications or surgery. Ophthalmology 2001;108:1943-53.

proteins such as vascular endothelial growth factor (VEGF). Treatment strategies that target pathologically elevated levels of VEGF are easy to understand: they try to block or reduce a known stimulus for the growth of CNV. Some early successes have been reported with anti-VEGF therapies. ${ }^{12}$

The waters are still muddy, though, when it comes to more fundamental, or at least earlier, steps in the process that leads an eye to develop AMD. What factors cause an ageing Bruch's membrane to become susceptible to fissure and invasion by CNV? Why do excess lipids accumulate to form drusen in some patients but not others? What disrupts the balance of pro-angiogenic and anti-angiogenic factors in the retinochoroid layers of the macula and promotes new vessel growth? These are 
only a few of the questions that have not yet been answered fully.

Enter thrombospondin. This glycoprotein was first described in 1971 and isolated in 1978 by researchers studying the mechanisms and regulation of the blood clotting process. ${ }^{34}$ It earned its name because it was released by platelets in response to treatment with thrombin. The protein was found to be an endogenous platelet participant in the haemagglutination process through its interactions with platelet bound fibrinogen. ${ }^{5}$ A multivalent molecule, it was found to have binding sites for many different molecules including collagen, fibronectin, fibrinogen, plasminogen, and calcium. ${ }^{6}$ Thrombospondin was also shown to be produced by other cell types including endothelial cells. ${ }^{7}$

Thrombospondin was thus understood early on to have an important role in the interactions of cells with other cells and with extracellular matrix. Immunostaining showed that thrombospondin was present in the interstices around multiple tissues in the body. Atherosclerotic lesions were found to have strong staining with anti-thrombospondin antibodies. ${ }^{8}$ This is especially relevant to studies of AMD because of its similarities to atherosclerosis in lipid accumulation and basement membrane damage.

Work on this molecule in the past few decades has progressed in many directions, since the molecule's precise roles have not yet been completely defined. One investigator described thrombospondin as a "protein in search of a function" and asked "what is this thrombospondin doing to the cells with which it interacts?" healing (in which thrombospondin is seen in healing but not healed wounds), and embryonic development (during which more thrombospondin staining is detected than in adults) are all areas that have pointed to a role for this protein in cell migration and adhesion. ${ }^{10}$

\section{Studies suggest that thrombospon- din is a critical regulator of angio- genesis in the eye-and much work remains to be done to understand its role fully}

Of especial relevance to AMD researchers was the discovery that thrombospondin has a regulatory role in angiogenesis. Initial work showed that the molecule was a homologue of an anti-angiogenic factor in hamsters, gp140. Thrombospondin was able to inhibit angiogenesis in vivo and prevent endothelial cell migration in vitro. ${ }^{11}$ These studies provided the first clue that thrombospondin released from a cell into the extracellular matrix might block neovascularisation by preventing endothelial cells from attaching to target structures in that space. Its role in angiogenesis is not as a simple suppressor, though, since thrombospondin can also induce migration and spreading of endothelial cells. ${ }^{12}$ Thus, thrombospondin has come to be known as a "modulator" of angiogenesis.

How might thrombospondin participate in the pathogenesis of AMD? The molecule is produced and secreted by retinal pigment epithelium (RPE) cells in culture and can be identified in the cytoplasm of RPE cells in a human eye section. ${ }^{13}$ These findings raised the possibility that RPE cells could contribute to a Bruch's membrane barrier against neovascular invasion from the choroid (CNV) by elaborating thrombospondin into the extracellular matrix. The next order of business was to begin to study thrombospondin in eyes both with and without AMD in order to understand how the balance of power changes in this disease.

In this issue of BJO ( $p$ 48) Uno and colleagues have taken this logical next step. Their simple yet crucial study contributes another piece to the angiogenesis in AMD puzzle. It introduces AMD to the thrombospondin literature and allows us to begin to apply all the knowledge about this protein that we have accumulated from other systems of the body.

In their study, Uno et al performed immunostaining of human eye sections with an anti-thrombospondin antibody. They compared 12 aged control eyes with 12 eyes with AMD. Their results support the notion that thrombospondin might have an anti-angiogenic function at Bruch's membrane: they found less thrombospondin in eyes with AMD than in matched controls. In addition, eyes with late AMD had less staining at Bruch's membrane than eyes with early AMD.

These investigators also sought an explanation for the lack of $\mathrm{CNV}$ in extramacular locations and therefore looked at the staining levels in the far periphery in addition to the macula. The finding that peripheral levels were lower than macular levels in all eyes, regardless of disease state, may merely be a function of Bruch's membrane being thinner in the periphery, as the authors suggest. It might also hint at a more complex relation between thrombospondin and angiogenesis in the eye than the main finding of the study, that there is less thrombospondin staining in eyes with AMD, might suggest.

Indeed, this complexity (or confusion) is highlighted by the observation that thrombospondin added to cultured RPE cells can actually increase the amount of VEGF released by these cells. ${ }^{14}$ On the other hand, thrombospondin-1 knockout mice develop retinal and choroidal neovascularisation in the setting of an insult such as uveitis, unlike normal mice. ${ }^{15}$ Taken together with the findings of Uno et al reported here, these and other studies suggest that thrombospondin is a critical regulator of angiogenesis in the eye-and that much work remains to be done to understand its role fully. One certainty is that the extracellular matrix and Bruch's membrane, where thrombospondin localises, are at centre stage for the action in the pathophysiology of AMD. Readers are certain to see more studies involving these important sites in the future.

Br J Ophthalmol 2006;90:5-6. doi: 10.1136/bjo.2005.079889

Correspondence to: Dr Jay M Stewart University of California, San Francisco, Department of Ophthalmology, 10 Koret Way, K301, San Francisco, CA 94143-0730, USA; ne62@yahoo.com

\section{REFERENCES}

1 Gragoudas ES, Adamis AP, Cunningham ET Jr, et al. Pegaptanib for neovascular age-related macular degeneration. N Engl J Med 2004;351:2805-16.

2 Michel S, Rosenfeld PJ, Puliafito CA, et al. Systemic bevacizumab (Avastin) for neovascular age-related macular degeneration: 12-week results of an uncontrolled open-label clinica study. Ophthalmology 2005; 112:1035-47.

3 Baenziger NL, Brodie GN, Majerus PW. A thrombin-sensitive protein of human platelet membranes. Proc Natl Acad Sci USA $1971 ; 68: 240-3$

4 Lawler JW, Slayter HS, Coligan JE. Isolation and characterization of a high-molecular weight glycoprotein from human blood platelets. J Biol glycoprotein from human bloor

5 Jaffe EA, Leung LLK, Nachman RL. Thrombospondin is the endogenous lectin of human platelets. Nature 1982;295:246-8.

6 Lawler J. The structural and functional properties of thrombospondin. Blood 1986;67:1 197-209.

7 Mosher DF, Doyle MJ, Jaffe EA. Synthesis and secretion of thrombospondin by cultured human endothelial cells. J Cell Biol 1982;93:343-8.

8 Wight TN, Raugi GJ, Mumy SM, et al. Light microscopic immunolocation of thrombospondin in human tissues. J Histochem Cytochem 1985;33:295-302.

9 Frazier WA. Thrombospondins. Curr Op Cell Biol 1991;3:792-9.

10 Mosher DF. Physiology of thrombospondin. Annu Rev Med 1990;41:85-97.

11 Good DJ, Polverini PJ, Rastinejad F, et al. A tumor suppressor-dependent inhibitor of angiogenesis is immunologically and functionally indistinguishable from a fragment of thrombospondin. Proc Natl Acad Sci USA 1990;87:6624-8.

12 Taraboletti G, Roberts D, Liotta LA, et al. Platelet thrombospondin modulates endothelial cell adhesion, motility, and growth: a potential angiogenesis regulatory factor. J Cell Biol 1990:111:765-72.

13 Miyajima-Uchida $\mathrm{H}$, Hayashi $\mathrm{H}$, Beppu $\mathrm{R}$, et al. Production and accumulation of thrombospondin1 in human retinal pigment epithelial cells. Invest Ophthalmol Vis Sci 2000;41:561-7.

14 Mousa SA, Lorelli W, Campochiaro PA. Role of hypoxia and extracellular matrix-integrin binding in the modulation of angiogenic growth factors secretion by retinal pigmented epithelial cells. J Cell Biochem 1999;74:135-43.

15 Zamiri P, Masli S, Kitaichi N, et al. Thrombospondin plays a vital role in the immune privilege of the eye. Invest Ophthalmol Vis Sci 2005;46:908-19. 